Nervenarzt 2015 $\cdot 86: 131-132$

DOI 10.1007/s00115-014-4077-8

Online publiziert: 30. Januar 2015

๑) Springer-Verlag Berlin Heidelberg 2015

\author{
A. Straube ${ }^{1} \cdot$ R. Dengler ${ }^{2}$ \\ ${ }^{1}$ Neurologische Klinik und Poliklinik \& Deutsches Schwindel- und Gleichgewichtszentrum \\ IFB, Ludwig-Maximilians-Universität München, Klinikum Großhadern, München \\ ${ }^{2}$ Neurologische Universitätsklinik, Medizinische Hochschule Hannover
}

\title{
Periphere Neurologie: kein Randgebiet in der täglichen klinischen Versorgung!
}

In den letzten Jahren sind die wissenschaftlichen Beiträge zur peripheren Neurologie, im Vergleich zur Zahl der Publikationen z. B. zu neurodegenerativen Erkrankungen oder Schlaganfall, in den Hintergrund gerückt. Wenn Beiträge zu Polyneuropathien veröffentlicht wurden, betrafen sie vorwiegend immunologische Formen. Im Kontrast dazu steht aber, dass in der ambulanten Versorgung peripherneurologische Fragen häufig im Vordergrund stehen.

Gerade Polyneuropathien (PNP) sind häufig und nehmen mit zunehmender Alterung der Bevölkerung weiter zu. Eine Schwierigkeit in der Beantwortung der Frage zur Häufigkeit durch epidemiologische Studien ist aber, dass diese sehr stark sowohl von den eingesetzten Erhebungsmethoden (Fragebogen, klinische oder elektrophysiologische Untersuchung) als auch von den untersuchten Bevölkerungsgruppen (nur Ältere, Patienten von Spezialambulanzen usw.) abhängt. In Süditalien wurde eine Prävalenz für PNP von 7\% auf der Grundlage einer Fragebogenuntersuchung gefunden [2]. In der Augsburger MONICA/KORA-Studie wurde eine PNP-Häufigkeit für Personen mit einer normalen Glukosetoleranz von $7,4 \%$ erhoben, für Probanden mit einer gestörten Glukosetoleranz von 13\% und mit manifestem Diabetes mellitus von $28,0 \%$ [4]. Patienten mit einem Diabetes mellitus Typ 1 sind etwas weniger häufig betroffen als Patienten mit einem Typ-2Diabetes (22,7\% zu 32,1\%; United Kingdom hospital study [3]). Die Häufigkeit hereditärer PNP (z. B. CMT1, 20-40/100 000 Einwohner) und Inzidenzen immu- nologischer Neuropathien (Guillain-Barré-Syndrom ca. 2/100 000 pro Jahr) sind dagegen um ein Vielfaches niedriger [2].

Eine Sonderform der Neuropathie sind die durch Druck bedingten Engpasssyndrome, auch diese sind häufig. So finden sich in der Bevölkerung Südschwedens bei $14,4 \%$ anamnestische Hinweise auf ein Karpaltunnelsyndrom und bei $3,8 \%$ ein klinisch sowie bei $4,9 \%$ ein elektrophysiologisch gesichertes [1].

Vor diesem Hintergrund sollen die Beiträge zu diesem Themenheft den modernen Stand von Klinik, Diagnostik und therapeutischen Ansätzen häufiger Formen peripherer Nervenstörungen vorstellen und auch auf wenig beachtete Zusammenhänge hinweisen.

\section{》) latrogene Nervenläsionen sind ein zahlenmäßig relevantes Problem}

In dem Beitrag von Schulte-Mattler und Grimm werden neben dem Karpaltunnelsyndrom auch weniger häufige Engpasssyndrome wie Incisura-scapulae-Syndrom, Kubitaltunnelsyndrom, Meralgia paraesthetica, „Thoracic-outlet“-Syndrom und Interosseus-anterior-Syndrom vorgestellt. Allen gemeinsam ist, dass häufig Schmerzen, die nicht immer dem Innervationsgebiet entsprechen, im Vordergrund stehen. Bedingt durch die Vielzahl invasiver Eingriffe sind traumatische, in diesem Falle iatrogene Nervenläsionen ein zahlenmäßig relevantes Problem. Im Beitrag von MüllerVahl wird der Frage nach dem therapeutischen Vorgehen und der Prognose trau- matischer Nervenläsionen nachgegangen. Ein Mechanismus, der vermutlich sowohl bei Druck- als auch bei traumatischen Läsionen zur Entwicklung des Nervenschadens beitragen kann, ist die Ischämie. Allein eine periphere arterielle Verschlusskrankheit kann schon zu einer Funktionsstörung und einer Neuropathie führen, was häufig nicht erkannt wird (Beitrag von Lang). Neben den traumatischen bzw. iatrogenen Nervenläsionen stellen die therapieassoziierten PNP und hier insbesondere die chemotherapiebedingten ein zunehmendes Problem dar. Böhmerle und Kollegen stellen die klinischen Besonderheiten der verschiedenen durch Chemotherapeutika bedingten PNP und die mögliche Einflussnahme auf die Häufigkeit von Neuropathien durch Änderung der Dosierungen, Frequenz der Applikation sowie Form der Gabe dar. Der letzte Beitrag von den Kollegen Doppler und Reimers beschäftigt sich mit der häufigsten spezifischen Neuropathie, der diabetischen Neuropathie und hier besonders mit den weniger bekannten Formen wie autonome Neuropathie, diabetische Radikulopathie und kraniale Mononeuropathien.

Natürlich können diese Beiträge nicht erschöpfend das Kapitel „periphere Neurologie" abdecken. Es erscheint den Herausgebern und Autoren aber notwendig und wichtig, den Blick auf dieses klinisch so relevante, in der Ausbildung des Nachwuchses aber zunehmend vernachlässigte Gebiet der Neurologie zu lenken, zumal es auch dort signifikante diagnostische und therapeutische Fortschritte gibt. Vielleicht gelingt es auch den einen oder anderen Leser für die periphere Neurolo- 
gie $\mathrm{zu}$ begeistern und anzuregen, dieses interessante Gebiet für sich zu entdecken.

Mit besten kollegialen Grüßen

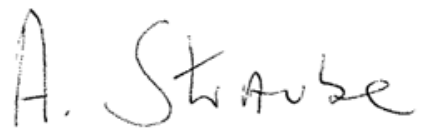

Prof. Dr. Andreas Straube

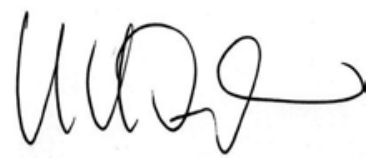

Prof. Dr. Reinhard Dengler

\section{Korrespondenzadressen}

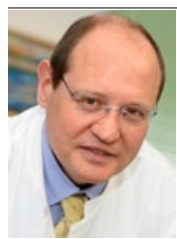

Prof. Dr. A. Straube

Neurologische Klinik und

Poliklinik \& Deutsches

Schwindel- und

Gleichgewichtszentrum

IFB, Ludwig-Maximilians-

Universität München,

Klinikum Großhadern

Marchioninistr. 15,

81377 München

Andreas.Straube@

med.uni-muenchen.de

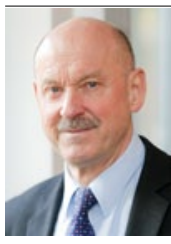

Prof. Dr. R. Dengler

Neurologische

Universitätsklinik,

Medizinische

Hochschule Hannover,

Carl-Neuberg-Str. 1

30625 Hannover

dengler.reinhard@

mh-hannover.de

Interessenkonflikt. A. Straube und R. Dengler geben an, dass kein Interessenkonflikt besteht.

\section{Literatur}

1. Atroshi I, Gummesson C, Johnsson R et al (1999) Prevalence of carpal tunnel syndrome in a general population. JAMA. 282(2):153-158

2. Martyn CN, Hughes RA (1997) Epidemiology of peripheral neuropathy. J Neurol Neurosurg Psychiatry 62(4):310-318

3. Young MJ, Boulton AJM, Macleod AF et al (1993) A multicentre study of the prevalence of diabetic peripheral neuropathy in the United Kingdom hospital clinic population. Diabetologia 36:150-154

4. Ziegler D, Rathmann W, Dickhaus T et al; KORA Study Group (2008) Prevalence of polyneuropathy in pre-diabetes and diabetes is associated with abdominal obesity and macroangiopathy: the MONICA/KORA Augsburg Surveys S2 and S3. Diabetes Care 31(3):464-469
M. Stöhr, R. Pfister

\section{Klinische Elektromyographie und Neurographie}

Lehrbuch und Atlas

Stuttgart: Kohlhammer Verlag 2014,

6. Auflage, 379 S., (ISBN 978-3-17-021473-6)

Der deutschsprachige Buchklassiker der klinischen Elektromyographie und Neurographie liegt nach mehr als 30 Jahren nach der 1 . Auflage nun in der 6 . Auflage vor. Unverändert ist es die große Stärke des Buches, sehr detailreich fast alle Untersuchungstechniken der klinischen Elektrophysiologie vorzustellen. Die mit den Methoden erhaltbaren Befunde werden auf der Basis der physiologischen Grundlagen und pathophysiologischen Veränderungen sowie ihrer klinischen Wertung eingehend besprochen. Nicht zuletzt hat das Buch schon immer eine Übertragung der Methodik der Befunde in die Diagnostik spezieller Krankheitsbilder ausgezeichnet, von häufigen alltäglichen Erkrankungen bis hin zu seltenen, ja raren.

Im Kapitel der Untersuchungstechniken werden die klassischen Methoden der Elektromyographie (EMG) der motorischen und sensiblen Neurographie vorgestellt. Darüber hinaus Untersuchungsmethoden mit sehr speziellen Anwendungen und besonderen Anforderungen an Technik und Untersuchungserfahrung. Es gehört zu den Stärken des Buches, diese selten genutzten Untersuchungstechniken und ihren Nutzen für die Differenzialdiagnostik schwieriger Situationen vorzustellen. Untersuchungsmethoden wie das Einzelfaser- oder Makro-EMG, die Hochvoltstimulation oder die Untersuchung des sympathischen Hautreflexes werden sicherlich in der klinischen Routine sehr selten angewendet werden und haben ihren Stellenwert in Zeiten immer weiter entwickelter Bildgebungstechniken von Nerven und Muskeln und immer weiter entwickelten Kenntnissen in der Labordiagnostik und nicht zuletzt der genetischen Diagnostik deutlich eingebüßt. So ist "leider" festzustellen, dass es den klinischen Elektrophysiologen, der mit sehr viel anatomischer und neurophysiologischer Kenntnis und viel Erfahrung mit einem breiten Werkzeug von Untersuchungstechniken diagnostische Entscheidungen trifft, kaum mehr geben wird.

Die Neurologie braucht den elektrophysiologischen Könner, der mit wenigen gezielten
Untersuchungen pragmatische diagnostisch wegweisende Entscheidungen treffen kann. Der immer noch große Wert des Buches liegt darin, sich nicht auf eine pragmatische evidenzbasierte Denkweise beschränkt zu haben.

Dennoch, der große Klassiker ist in die Jahre gekommen. Dies liegt sicherlich zum großen Teil daran, dass die klinische Elektrophysiologie, obwohl unverzichtbarer Teil der neurologischen Diagnostik, in weiten Teilen keine gute evidenzbasierte statistisch geprüfte Basis hat. Dies liegt aber auch daran, dass das Buch, vielleicht leider, vielleicht aber auch zum Glück, keine fertigen Untersuchungskonzepte vorlegt. Dem Anfänger und dem wenig Erfahrenen, demjenigen der Basiswissen erwerben will und einfache Handlungsanweisungen erlernen möchte, wird es mit dem Buch schwer werden. Für denjenigen, dem es um seine Breite an klinisch elektro-/neurophysiologischer Methodik und Anwendung geht, ist das Buch nach wie vor unverzichtbar. Leider ist der Neuauflage der Umstieg aus analoger in die digitale Zeit nicht gelungen. Vielleicht war das auch gar nicht Ziel. Aber wer weiß denn schon noch, was eine Kippgeschwindigkeit ist, und wer hat noch teflonisolierte Nadelelektroden, die sterilisiert werden müssen, zur Verfügung? Neben vielen Abbildungen, die sehr detailreich gezeichnet und von hohem didaktischem Wert sind, enthält das Buch viele Abbildungen aus der analogen Zeit, die die Informationen nicht mehr gut transportieren können. Leider sind immer noch viele Abbildungen in englischer Sprache beschriftet. Aus der Sicht eines Pragmatikers der klinischen Elektroneurophysiologie wirkt das Konzept des Buches nicht mehr zeitgemäß. Der große Klassiker der klinischen elektroneurophysiologischen Diagnostik ist in die Jahre gekommen. Darin liegen ein großer Vorzug und eine große Güte des Buches, weil die "alte" Sicht nicht verloren gegangen ist. Ich würde mir eine sehr grundsätzliche Revision des Buches wünschen mit dem Ziel, das darin enthaltene breite Wissen nicht zu verlieren, es auf aktuelle Technik zu modernisieren und den pragmatischen Notwendigkeiten diagnostischer Entscheidungen anzupassen.

H. Buchner (Recklinghausen) 